\title{
Cerebral deep venous thrombosis and COVID-19: case report
}

\author{
Christian Hoelscher, MD, Ahmad Sweid, MD, Ritam Ghosh, MD, Fadi Al Saiegh, MD, \\ Kavantissa M. Keppetipola, MD, Christopher J. Farrell, MD, Jack Jallo, MD, PhD, \\ Pascal Jabbour, MD, Stavropoula Tjoumakaris, MD, M. Reid Gooch, MD, \\ Robert H. Rosenwasser, MD, and Syed O. Shah, MD, MBA
}

\author{
Department of Neurological Surgery, Thomas Jefferson University Hospital, Philadelphia, Pennsylvania
}

\begin{abstract}
Herein, the authors present the case of a 54-year-old male diagnosed with coronavirus disease 2019 (COVID-19) during a screening test. The patient was asked to self-isolate at home and report with any exacerbations of symptoms. He presented later with pneumonia complicated by encephalopathy at days 14 and 15 from initial diagnosis, respectively. MRI of the brain showed bithalamic and gangliocapsular FLAIR signal abnormality with mild right-sided thalamic and periventricular diffusion restriction. A CT venogram was obtained given the distribution of edema and demonstrated deep venous thrombosis involving the bilateral internal cerebral veins and the vein of Galen. CSF workup was negative for encephalitis, as the COVID-19 polymerase chain reaction (PCR) test and bacterial cultures were negative. A complete hypercoagulable workup was negative, and the venous thrombosis was attributed to a hypercoagulable state induced by COVID-19. The mental decline was attributed to bithalamic and gangliocapsular venous infarction secondary to deep venous thrombosis. Unfortunately, the patient's condition continued to decline, and care was withdrawn.
\end{abstract}

https://thejns.org/doi/abs/10.3171/2020.5.JNS201542

KEYWORDS COVID-19; SARS-CoV-2; central nervous system; cerebrovascular disease; hypercoagulable state; deep venous thrombosis; vascular disorders; infection

$\mathrm{O}$ N March 11, 2020, the World Health Organization declared that coronavirus disease 2019 (COVID-19), caused by severe acute respiratory syndrome coronavirus 2 (SARS-CoV-2), is a pandemic. ${ }^{1}$ The virus has a high reproduction number (2.0-2.5) allowing it to infect a large number of the world population. ${ }^{2}$ The primary organ affected is the respiratory system; however, neurological manifestations have been reported. ${ }^{3}$ Herein, we describe a case of COVID-19 infection with pulmonary manifestations and encephalopathy due to cerebral venous infarction secondary to deep venous thrombosis.

\section{Case Report}

History and Examination

A 54-year-old male, with a medical history notable only for hypertension, tested positive for COVID-19 at another institution during a screening examination conducted due to close contact with a COVID-19-positive individual on April 5, 2020. SARS-CoV-2 infection was confirmed by reverse transcriptase polymerase chain reaction (RT-PCR) assay. The patient was asked to self-quarantine; however, 9 days after testing positive, he presented with shortness of breath and cough. The patient was diagnosed with pneumonia and started on oxygen, ceftriaxone, and azithromycin. The patient's mental status declined (day 15 after the initial diagnosis), for which he required mechanical ventilation. CT imaging of the brain showed bithalamic and gangliocapsular hypodensity with mass effect and approximately $6 \mathrm{~mm}$ of rightward midline shift, as well as prominence of the bilateral temporal horns. Subsequent MRI of the brain showed bithalamic and gangliocapsular FLAIR signal abnormality with mild right-sided thalamic and periventricular diffusion restriction. The temporal horns were prominent with evidence of transependymal flow. He was transferred to our institution for further management.

ABBREVIATIONS COVID-19 = coronavirus disease 2019; CSF = cerebrospinal fluid; EVD = external ventricular drain; ICP = intracranial pressure; SARS-CoV-2 = severe acute respiratory syndrome coronavirus 2.

SUBMITTED April 29, 2020. ACCEPTED May 29, 2020.

INCLUDE WHEN CITING Published online September 4, 2020; DOI: 10.3171/2020.5.JNS201542. 

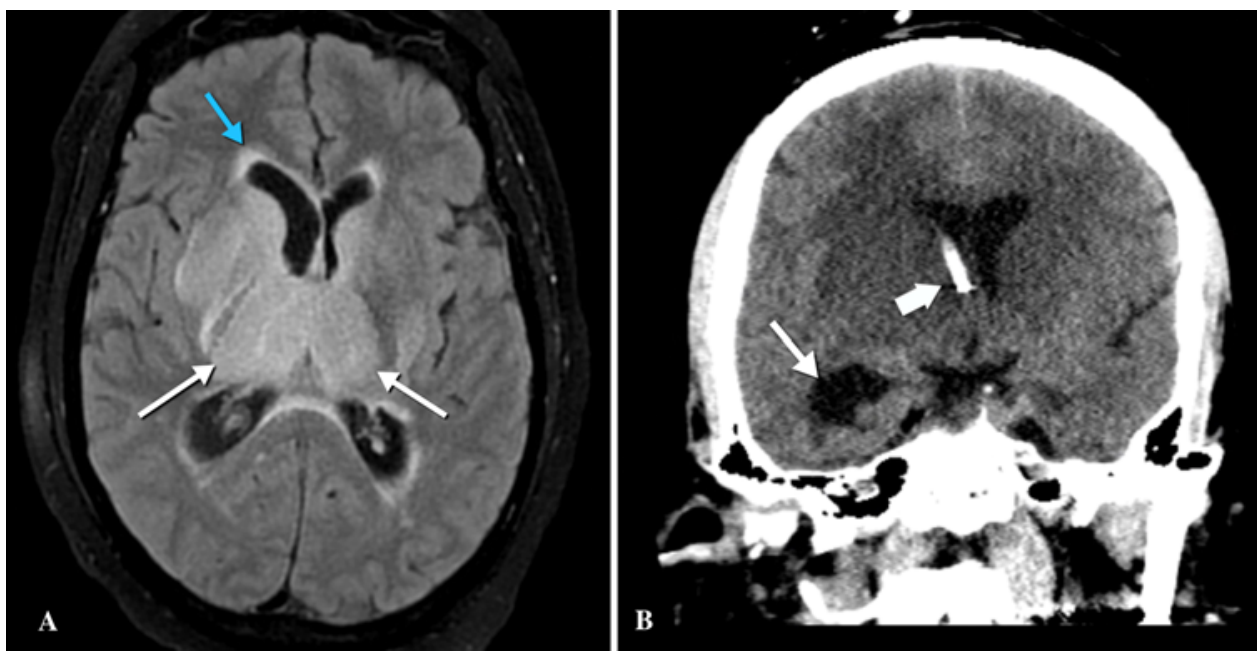

FIG. 1. A: Axial FLAIR MRI showing hyperintense signal in the bilateral thalami (white arrows) and basal ganglia region (worse on the patient's right). Note the transependymal flow at the right frontal horn (blue arrow). B: Coronal CT scan showing the EVD in the right frontal horn (thick arrow). Note the dilated right temporal horn (small arrow) indicating hydrocephalus. Figure is available in color online only.

Upon admission to Thomas Jefferson University Hospital, the patient was obtunded, opening his eyes and grimacing only to deep noxious stimulation, localizing his bilateral upper extremities weakly, and withdrawing his bilateral lower extremities (Glasgow Coma Scale [GCS] E2V2M5). His pupils were equal and reactive, and protective brainstem reflexes were intact. Auscultation of his lungs revealed coarse breath sounds bilaterally, consistent with his known COVID-19 pulmonary pathology. Given

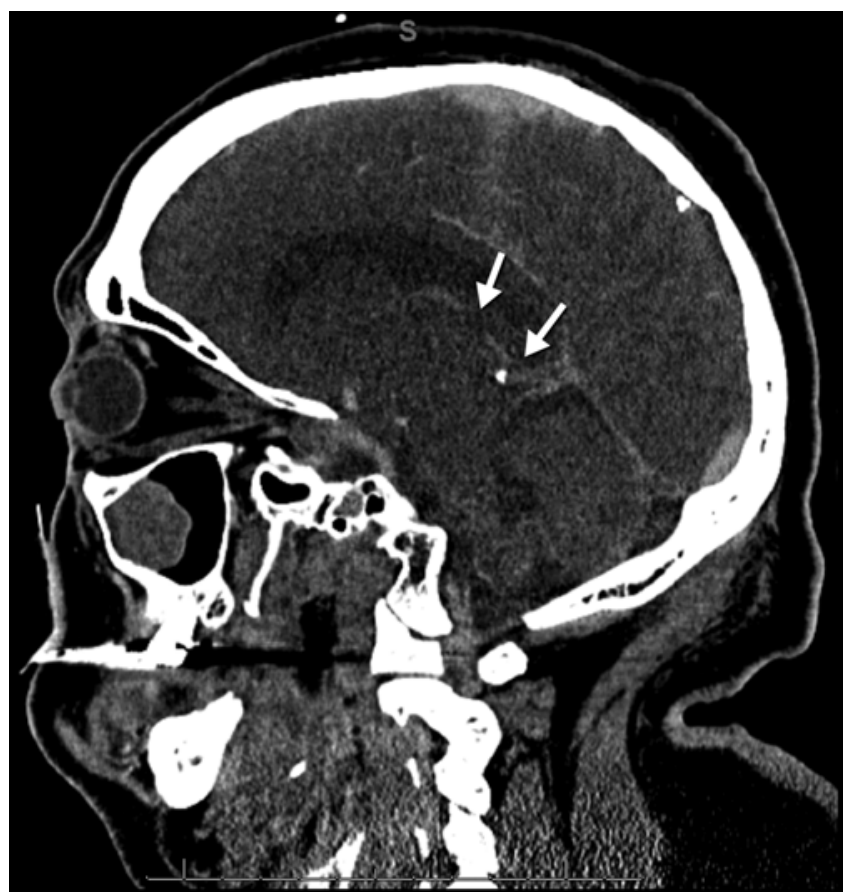

FIG. 2. Sagittal CT venogram showing filling defects in the internal cerebral vein (top arrow) and vein of Galen (bottom arrow) consistent with thrombosis. his poor mental status and concern for obstructive hydrocephalus, a right external ventricular drain (EVD) was placed for cerebrospinal fluid (CSF) diversion, intracranial pressure (ICP) monitoring, and CSF studies. The CSF opening pressure was $30 \mathrm{~cm} \mathrm{H}_{2} \mathrm{O}$ (Fig. 1). A CT venogram, obtained given the distribution of edema, demonstrated deep venous thrombosis involving the bilateral internal cerebral veins and the vein of Galen (Fig. 2), as well as progressive deep cerebral edema. Pertinent laboratory findings on admission to the intensive care unit are summarized in Table 1. Notable findings included elevated levels of D-dimer, erythrocyte sedimentation rate, lactate dehydrogenase, and serum ferritin. A complete hypercoagulable workup did not identify the provocative cause for the venous thrombosis, and the venous thrombosis was attributed to a hypercoagulable state induced by COVID-19 (antithrombin III, antiphospholipid antibodies, etc.). Also, CSF studies showed a markedly increased protein content and white blood cell count, initially suggesting the possibility of COVID-19 encephalitis. However, SARS-CoV-2 RNA was not detected in the CSF, and bacterial cultures remained negative.

\section{Treatment and Posttreatment Course}

The patient was started on hydroxychloroquine for COVID-19 treatment. A heparin infusion was initiated given the cerebral deep venous thrombosis, and a $3 \%$ hypertonic saline infusion was administered given concerns over increasing mass effect due to cerebral edema. Serial CT scans revealed a punctate focus of hemorrhage in the right basal ganglia that remained stable. The patient had an episode of ICP crisis with a dilated right pupil that was responsive to $23.4 \%$ hypertonic saline administration. His right frontal ventricular drain was not draining CSF at this point. A subsequent CT scan showed the ventricle collapsed around the catheter tip, a dilated left ventricle, and progressive deep cerebral edema. The heparin infusion was temporarily held and a left-sided ventricular drain 
TABLE 1. Demographic and clinical characteristics and laboratory findings

\begin{tabular}{|c|c|}
\hline Factor & Description \\
\hline \multicolumn{2}{|l|}{ Demographic characteristics } \\
\hline Age (yrs) & 54 \\
\hline Sex & M \\
\hline \multicolumn{2}{|l|}{ Initial findings } \\
\hline Medical history & Hypertension \\
\hline Symptoms at disease onset & Shortness of breath, cough \\
\hline Imaging features & Lt lower lobe pneumonia \\
\hline $\begin{array}{l}\text { Treatment before admission to } \\
\text { ICU }\end{array}$ & $\mathrm{O}_{2}$, ceftriaxone, \& azithromycin \\
\hline $\begin{array}{l}\text { No. of days from disease onset to } \\
\text { thrombotic events }\end{array}$ & 15 \\
\hline \multicolumn{2}{|l|}{ Findings on admission to ICU } \\
\hline No. of days since disease onset & 15 \\
\hline Disease severity & Severe \\
\hline \multicolumn{2}{|l|}{ Laboratory findings } \\
\hline WBC (B/L) & 7.8 \\
\hline \multicolumn{2}{|l|}{ Differential count (B/L) } \\
\hline Total neutrophils & 6.42 \\
\hline Total lymphocytes & 1.09 \\
\hline Total monocytes & 0.17 \\
\hline Platelet count (B/L) & 372 \\
\hline Hemoglobin (g/dl) & 14.4 \\
\hline Albumin (g/dl) & 3.7 \\
\hline ALT (IU/L) & 67 \\
\hline AST (IU/L) & 44 \\
\hline $\mathrm{LDH}(\mathrm{IU} / \mathrm{L})$ & 382 \\
\hline Creatinine (mg/dl) & 1.06 \\
\hline Creatine kinase (IU/L) & 190 \\
\hline $\mathrm{EGFR}\left(\mathrm{ml} / \mathrm{min} / 1.73 \mathrm{~m}^{2}\right)$ & $>60$ \\
\hline hs-cTnT (ng/L) & 14 \\
\hline Prothrombin time (sec) & 11.8 \\
\hline aPTT (sec) & 25 \\
\hline Fibrinogen (mg/dl) & 429 \\
\hline Antithrombin III (\%) & 99 \\
\hline D-dimer (ng/ml) & 3151 \\
\hline Serum ferritin (ng/ml) & 508 \\
\hline Procalcitonin (ng/ml) & 0.2 \\
\hline High-sensitivity CRP (mg/dl) & 0.60 \\
\hline $\mathrm{ESR}(\mathrm{mm} / \mathrm{hr})$ & 47 \\
\hline \multicolumn{2}{|l|}{ CSF studies } \\
\hline 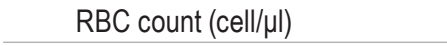 & 401 \\
\hline WBC (cell/ $/ \mu \mathrm{l})$ & 10 \\
\hline Glucose (mg/dl) & 38 \\
\hline Protein (mg/dl) & 1104 \\
\hline
\end{tabular}

CONTINUED IN NEXT COLUMN »
» CONTINUED FROM PREVIOUS COLUMN

TABLE 1. Demographic and clinical characteristics and laboratory findings

\begin{tabular}{ll}
\hline \multicolumn{1}{c}{ Factor } & \multicolumn{1}{c}{ Description } \\
\hline $\begin{array}{l}\text { Findings on admission to ICU } \\
\text { (continued) }\end{array}$ & \\
\hline \multicolumn{1}{c}{ Imaging features } & Encephalitis w/ midline shift; \\
& venous infarct involving \\
& bithalamic \& basal ganglia; \\
& deep venous thrombosis \\
& involving straight sinus, \\
& vein of Galen, bilat internal \\
& cerebral veins, \& rt basal \\
& vein of Rosenthal \\
\hline
\end{tabular}

$\mathrm{ALT}=$ alanine aminotransferase; $\mathrm{aPTT}=$ activated partial thromboplastin time; $\mathrm{AST}=$ aspartate aminotransferase $; \mathrm{B} / \mathrm{L}=$ billion per liter; $\mathrm{CRP}=\mathrm{C}$-reactive protein; EGFR = estimated glomerular filtration rate; ESR = erythrocyte sedimentation rate; hs-cTnT = high-sensitivity cardiac troponin $\mathrm{T} ; \mathrm{LDH}=$ lactate dehydrogenase; RBC = red blood cell; $\mathrm{WBC}=$ white blood cell. Bold values indicate higher than normal range.

was placed. These measures allowed for transient control of ICP, but unfortunately he developed progressive bilateral deep venous infarctions involving critical structures and sustained elevated ICPs, and care was withdrawn on the 9th day of the hospital stay.

\section{Discussion}

Neurological manifestations caused by COVID-19 range from mild to severe symptoms. Several published reports have noted severe headache, anosmia, encephalopathy, encephalitis, meningitis, and acute cerebrovascular disease such as ischemic and hemorrhagic stroke. ${ }^{3-5}$ The coronavirus family has previously shown the ability to directly infect the central nervous system, and this may be the cause of some such presentations. Additionally, some severe presentations are thought to be at least partly attributable to an unusually intense inflammatory response, which has been widely reported in pulmonary tissue. ${ }^{3}$ Such a response may predispose to a hypercoagulable state, putting patients at risk for stroke due to either arterial occlusion or venous congestion. There are numerous reports documenting an increased risk of thromboembolic events in COVID-19-positive patients. ${ }^{6,7}$ Cerebral infarcts secondary to antiphospholipid antibodies in COVID-19 patients have also been reported. ${ }^{7}$ It is paramount to keep a high index of suspicion of venous thrombosis when faced with an encephalitis picture in the setting of COVID-19. The management is completely different, in which anticoagulation may improve outcomes.

\section{Conclusions}

SARS-CoV-2 may induce a hypercoagulable state causing cerebral venous thrombosis. The radiological features may share similarities with several differential diagnoses, including encephalitis. It is paramount to perform a complete workup because the management is completely 
different, and this affects the patient's outcome. It is crucial to account for such manifestations when managing patients with COVID-19.

\section{References}

1. Bedford J, Enria D, Giesecke J, et al. COVID-19: towards controlling of a pandemic. Lancet. 2020;395(10229):10151018.

2. Akhmerov A, Marbán E. COVID-19 and the heart. Circ Res. 2020;126(10):1443-1455.

3. Mao L, Jin H, Wang M, et al. Neurologic manifestations of hospitalized patients with coronavirus disease 2019 in Wuhan, China. JAMA Neurol. 2020;77(6):683-690.

4. Moriguchi T, Harii N, Goto J, et al. A first case of meningitis/encephalitis associated with SARS-Coronavirus-2. Int $J$ Infect Dis. 2020;94:55-58.

5. Poyiadji N, Shahin G, Noujaim D, et al. COVID-19-associated acute hemorrhagic necrotizing encephalopathy: CT and MRI features. Radiology. 2020;296(2):E119-E120.

6. Connors JM, Levy JH. Thromboinflammation and the hypercoagulability of COVID-19. J Thromb Haemost. 2020;18(7):1559-1561.
7. Zhang Y, Xiao M, Zhang S, et al. Coagulopathy and antiphospholipid antibodies in patients with Covid-19. N Engl J Med. 2020;382(17):e38.

\section{Disclosures}

Dr. Jabbour is a consultant for Medtronic and MicroVention. Drs. Tjoumakaris and Gooch are consultants for Stryker.

\section{Author Contributions}

Conception and design: Al Saiegh. Acquisition of data: Ghosh, Keppetipola. Drafting the article: Hoelscher, Sweid, Ghosh, Al Saiegh. Critically revising the article: Shah, Hoelscher, Sweid, Ghosh. Reviewed submitted version of manuscript: Shah, Farrell, Jallo, Jabbour, Tjoumakaris, Gooch, Rosenwasser. Approved the final version of the manuscript on behalf of all authors: Shah. Administrative/technical/material support: Keppetipola, Rosenwasser.

\section{Correspondence}

Syed Omar Shah: Thomas Jefferson University Hospital, Philadelphia,PA.syed.shah@jefferson.edu. 